\title{
Quando i bruciatai fiorentini divennero buzzurri
}

\author{
Pietro Trifone
}

PUBBLICATO: 07 GENNAIO 2020

\section{Quesito:}

Una lettrice ci scrive chiedendoci se sia condivisibile l'etimologia proposta da Francesco Zambaldi nel suo Vocabolario Etimologico Italiano che fa derivare il termine buzzurro dal tedesco Putzer.

\section{Quando i bruciatai fiorentini divennero buzzurri}

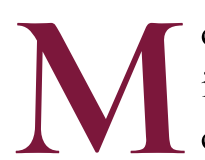
entre burino è una parola di sicura origine romanesca, buzzurro è invece un vocabolo importato subito dopo il I87o nella nuova e definitiva capitale d'Italia da Firenze, dove circolava da tempo - probabilmente prima dei più antichi esempi finora noti, che risalgono all'inizio dell'Ottocento - per designare i castagnai ambulanti svizzeri dei Cantoni del Ticino e dei Grigioni che d'inverno lasciavano le loro montagne e venivano a vendere caldarroste (fiorentinamente bruciate) nella città. Dopo il I 865, nella breve stagione di Firenze capitale, l'epiteto di buzzurro è applicato in senso spregiativo ai funzionari e ai militari piemontesi che si trasferiscono in massa, con le rispettive famiglie, sulle rive dell'Arno; tanto che nel i87o Pietro Fanfani registra nelle Voci e maniere del parlare fiorentino (I870) il recente sviluppo semantico, aggiungendo alla precedente e più specifica accezione del vocabolo quella di "Uomo zotico, sgarbato, e di poca creanza". Proprio a Roma, tuttavia, il termine trovò una straordinaria cassa di risonanza, come risulta dal suo massiccio impiego in senso antiunitario e antiliberale sulle colonne della "Civiltà cattolica", organo ufficiale dello schieramento papalino.

Se appare chiaro l'intento discriminatorio racchiuso fin dall'inizio nella separazione lessicale tra $i$ buzzurri forestieri e i preesistenti bruciatai fiorentini - categoria colpita a sua volta da un generale discredito -, non è facile determinare con sicurezza il punto di partenza del processo che ha indotto a chiamare in quel modo piuttosto che in un altro i venditori ambulanti giunti a Firenze da terre distanti, non solo in senso geografico. Risultano scarsamente plausibili le spiegazioni etimologiche che collegano buzzurro a buzzo 'ventre, pancia' o al tedesco Putzer 'pulitore, spazzacamino', non solo per la debolezza delle relazioni semantiche tra i termini che dovrebbero essere imparentati tra loro, ma anche per ragioni strutturali: da questo punto di vista, se la distanza tra Putzer e buzzurro sembra davvero eccessiva, la stessa trafila buzzo > buzzurro appare improbabile, data la difficoltà di supporre un fecondo innesto diretto sul toscano buzzo di un suffisso come-urro, sostanzialmente improduttivo nelle varietà linguistiche della Toscana.

Un'ipotesi stimolante, sebbene non priva di difficoltà, è stata avanzata da Ottavio Lurati e poi accolta nel dizionario etimologico di Alberto Nocentini: buzzurro sarebbe una retroformazione da buzza(r)rone, buzzur(r)one, forme attestate in zone diverse (buzzeron ebuzzaron nei dialetti settentrionali, buzzarruni e buzzurruni in siciliano), e corrispondenti al toscano buggerone e al romanesco buggiarone, il cui primo significato è quello di 'sodomita'. Il punto di partenza di tutta la ramificata famiglia lessicale sarebbe costituito infatti dal latino tardo Bügerus, variante di Būlgarus, propriamente 'Bulgaro', passato a indicare l'eretico e quindi, con ulteriore sviluppo offensivo, il sodomita (sull'avventurosa vicenda di questo vocabolo si veda ora il bel libro di Enrico Testa, Bulgaro. Storia di una parola malfamata, Bologna, Il Mulino, 2019). Sulla bocca dei parlanti fiorentini il 
settentrionale buz(z)aron sarebbe divenuto buzzarone o meglio buzzerone; da quest'ultima forma doveva quindi ricavarsi un buzzurone, forse in seguito a un'assimilazione vocalica progressiva $u-e>u$-u, peraltro piuttosto atipica; al raddoppiamento della $r$, come a quello della $z$, potrebbe aver contribuito il modello di azzurro, sostenuto dalla mancanza in toscano di parole terminanti in -zuro.

Si tratta di sviluppi problematici (specialmente il passaggio $e>u$ ), ma in linea teorica non impossibili, considerato che l'adattamento di un prestito avviene di solito nella lingua d'arrivo e tende a produrre involontarie alterazioni della forma di partenza. Non si può fare a meno di rilevare, peraltro, che la supposta sequenza di mutamenti intervenuti non è sorretta da testimonianze toscane, dirette o indirette, delle fasi intermedie costituite da buzzarone, buzzerone e soprattutto da buzzurone, buzzurrone (forme, queste ultime, prive di riscontri significativi anche in area settentrionale). Quanto al siciliano buzzurruni, poi, la sua attestazione tarda fa pensare a una semplice variante locale dellitaliano comune buzzurrone, accrescitivo di buzzurro di cui abbondano gli esempi fin dagli anni Settanta dell'Ottocento. Del resto non sarebbe facile giustificare la penetrazione nel Granducato di Toscana di meridionalismi tanto decentrati e peculiari.

Le nebbie si diraderebbero se si trovasse già bell'e pronta nei dialetti settentrionali la parola buzzurro, o altra di forma simile, oltre che di significato riferibile o collegabile ai caldarrostai o bruciatai italosvizzeri di Firenze. A questo proposito, Mauro Braccini ha notato la singolare somiglianza di buzzurro con un vocabolo attestato nel Cantone dei Grigioni, precisamente in Val Bregaglia: büzùr 'ronzio, fruscio, brusio, mormorio'. Lo stesso studioso si è chiesto quindi se l'epiteto non abbia designato all'origine un 'balbuziente', un 'barbaro' proveniente da quelle zone. Il punto debole dell'ipotesi risiede nell'improbabilità che i venditori di caldarroste parlassero di ronzio, fruscio, brusio o mormorio cosi spesso da spingere i fiorentini, che non potevano conoscere il termine büzúr per altra via, a usarlo a loro volta per identificarli.

Sembra più motivato, semmai, un collegamento con il tipo largamente conosciuto in area settentrionale brüzur, brüzur brüsur, brusour 'bruciore', per la strettissima attinenza tra il bruciore e le bruciate. Da un lato troviamo la locuzione brüsur a la lengua a Pedrinate, il centro più meridionale del Canton Ticino; dall'altro il dizionario di Policarpo Petrocchi (I887-I89I) registra un invitante appello dei buzzurri a comprare le bruciate: "Bruciate calde, e fumano! Gridano i buzzurri". La risagomatura di brüzur o brüsur in buzzurro, probabilmente ispirata o favorita dal modello di azzurro, ha condotto alla dissimilazione regressiva della prima $r$, che semplifica il nesso biconsonantico $b r$; nello stesso tempo ha causato il raddoppiamento della $r$ finale, per esigenze di struttura sillabica. Resta tuttavia da verificare se i primi venditori italo-svizzeri di caldarroste in giro per Firenze fossero inclini a dire qualcosa come brüzur o brüsur, magari per avvertire gli acquirenti del pericolo di scottarsi la lingua o le mani, e se proprio quello strano richiamo, che ai fiorentini poteva forse risultare oscuro anche per la pronuncia marcata di quei bruciatai, sia divenuto con piccole modifiche il marchio di chi lo emetteva.

Tirando le somme, direi che l'etimologia di buzzurro continua a essere incerta, nonostante l'abbondanza delle informazioni riguardanti diverse tappe del suo percorso evolutivo e la possibilità di tracciare più di una linea di sviluppo. I dubbi residui potrebbero essere risolti dalla scoperta di attendibili testimonianze d'epoca, o almeno di indizi dal forte valore probatorio, che confermino una delle ipotesi messe in campo, o eventualmente permettano di aprire una nuova valida direzione di ricerca. 


\section{Cita come:}

Pietro Trifone, Quando i bruciatai fiorentini divennero buzzurri , "Italiano digitale", 2020, XII, 2020/1 (gennaio-marzo)

DOI: $10.35948 / 2532-9006 / 2020.3314$

Copyright 2020 Accademia della Crusca

Pubblicato con licenza creative commons CC BY-NC-ND 